\title{
FDG PET vascular imaging in IgG4-RD: Potential and challenges
}

\author{
Darko Pucar, $M D, P h D,{ }^{a}$ and Monique Hinchcliff, $M D, M S^{b}$ \\ a Section of Nuclear Medicine, Department of Radiology and Biomedical Imaging, Yale \\ University School of Medicine, New Haven, CT \\ b Department of Rheumatology, Allergy and Immunology, Yale University School of Medicine, \\ New Haven, CT
}

Received Nov 28, 2021; accepted Nov 29, 2021

doi: $10.1007 / \mathrm{s} 12350-021-02887-1$

\section{See related article, pp. $2920-2933$}

IgG4-related disease (IgG4-RD) is a rare group of systemic chronic fibroinflammatory disorders characterized by inflammatory tumors in body cavities and organs, with consequent organ dysfunction. ${ }^{1-3}$ Inflammatory tumors contain dense lymphoplasmacytic infiltrates of IgG4-positive plasma cells and characteristic storiform (whorled-pattern) fibrosis. ${ }^{1}$ The clinical presentation depends on the organs involved with the retroperitoneum and pancreas being most common and whether dysfunction is synchronous or metachronous. Cardiovascular organs involved are the aorta and its branches, cardiac valves, pericardium, myocardium, and pulmonary circulation. Involved spaces and organs also include salivary glands, orbits, paranasal sinuses, pharynx, thyroid, pituitary, meninges, lungs, pleura, mediastinum, breasts, liver, biliary system, gallbladder, mesentery, kidneys, prostate, vas deferens, scrotum, lymph nodes, skin, and nerves. ${ }^{1-3}$

The majority of patients are men greater than 50 years old. The presentation is typically subacute and related to the organs involved, while fever and C-reactive protein elevation are unusual. Allergic features are common, such as chronic sinusitis, asthma, and eczema. Many patients are diagnosed incidentally from analysis

Reprint requests: Darko Pucar, MD, PhD, Section of Nuclear Medicine, Department of Radiology and Biomedical Imaging, Yale University School of Medicine, 333 Cedar Street, New Haven, CT 06511; darko.pucar@yale.edu

J Nucl Cardiol 2022;29:2934-7.

$1071-3581 / \$ 34.00$

Copyright ( 2021 The Author(s) under exclusive licence to American Society of Nuclear Cardiology of pathologic specimens and imaging exams. Although the medical literature often presents IgG-RD as a mild condition, major organ damage often occurs in the chronic setting. For example, IgG4-related aortitis can cause severe complications, such as aneurysms and dissections. ${ }^{3,4}$ The diagnosis is made based on a combination of clinical, laboratory, imaging, and pathologic parameters, such as 2011 comprehensive diagnostic criteria for IgG4-RD proposed by Umehara, ${ }^{5}$ in the absence of malignancy or other diseases that could explain the presentation.

Although most patients have elevated serum IgG4 levels, the concentration is very variable. Moreover, $30 \%$ of patients have normal serum IgG4 despite diagnostic pathologic findings. ${ }^{6}$ The utility of serial IgG4 measurement is controversial. Whereas, IgG4 concentration typically declines but can remain elevated after glucocorticoid treatment, ${ }^{6}$ persistently elevated serum IgG4 levels occurs in about $70 \%$ of patients with lasting remission. ${ }^{7}$ Conversely, $10 \%$ of patients experience relapsing disease despite normal serum $\mathrm{IgG} 4{ }^{7}{ }^{7}$ These findings strongly suggest the potential value of alternative biomarkers including imaging for assessing IgG4RD activity.

Treatment, required in most patients, aims to prevent serious organ dysfunction. ${ }^{3,8}$ Some patients (e.g., those with asymptomatic lymphadenopathy) can be actively surveilled without treatment. Conversely, urgent treatment may be required in patients with the involvement of the aorta, pancreas, biliary tree, kidneys, retroperitoneum, mesentery, mediastinum, and meninges. Imaging may play a critical role in treatment decision. Glucocorticoids (prednisone $.6 \mathrm{mg} / \mathrm{kg}$ once daily or in divided doses) is typically first-line therapy for IgG4-RD ${ }^{3,8}$ and usually result in an adequate and prompt response. The initial steroid dose is usually maintained over 2-4 weeks and then gradually tapered 
over 3-6 months based on the response. Rituximab is indicated for patients with recurrent or refractory severe disease, while other steroid sparing agents (e.g., azathioprine, mycophenolate mofetil, or methotrexate) and longer-term steroid treatment may be indicated in some patients. ${ }^{3}$ Imaging may be a useful approach to guide treatment decisions until a circulating biomarker is identified.

The IgG4-RD imaging features are variable and often not specific enough to allow distinction from mimickers such as malignancy and other autoimmune diseases by imaging alone. ${ }^{3,9}$ Typically, patients will initially undergo conventional imaging with computed tomography (CT) or magnetic resonance imaging (MRI) of the affected region(s). ${ }^{9}$ Because approximately $60 \%$ of patients will have multi-organ involvement at the time of diagnosis, chest and abdominal imaging at a minimum should be considered to screen for asymptomatic organ involvement. ${ }^{9}$ 18-Fluorodeoxyglucose positron emission tomography/computed tomography (FDG PET/CT) has been shown to be an effective imaging modality for IgG4-RD evaluation. ${ }^{10-13}$ The distinct advantages of FDG PET/CT include widespread anatomic coverage (skull vertex or base to mid thighs) and an ability to assess IgG4 lesional activity. Thus, FDG PET/CT can be used for diagnosis, biopsy guidance, staging, treatment response assessment, and restaging. ${ }^{10-13} \mathrm{CT}$ performed with PET is usually a limited low-radiation dose exam for anatomic localization and attenuation correction and thus is non-diagnostic for independent interpretation. In order to improve diagnostic accuracy, diagnostic contrast-enhanced CT (CECT) can be performed separately and co-registered with PET or alternatively CECT and PET can be performed simultaneously. ${ }^{13}$ Moreover, PET/MRI is gradually becoming more widely available, ${ }^{14}$ which is important since MRI may be superior over CT in some clinical scenarios, such as skull base and nervous system imaging. In spite of its utility, FDG PET/CT is criticized for its radiation exposure, high cost, limited availability, and insurance coverage challenges. ${ }^{9}$

Zhang and colleagues conducted a prospective trial in China (NCT01665196) and characterized FDG PET/ CT findings in 35 patients with IgG4-RD based upon the Umehara diagnostic criteria. ${ }^{12}$ Additionally, 29 patients underwent repeated PET scans following 2-4 weeks of initial prednisone treatment $(40-50 \mathrm{mg} /$ day $)$. The FDG PET/CT scans were performed according to standard oncologic protocol with imaging 1-hour post-injection. At baseline, all patients had positive PET scans and 97\% had multi-organ disease on PET. Moreover, PET/CT imaging revealed that $71 \%$ had more lesions identifiable on PET compared to conventional imaging and in $20 \%$ the lesional biopsy site was changed to a more accessible location. The most common diseased sites were lymph nodes $(86 \%)$, salivary glands $(66 \%)$, and pancreas $(51 \%)$, while aortic involvement occurred in $23 \%$ of patients. Based on PET Response Criteria in Solid Tumors (PERCIST) 1.0, the authors observed complete and partial metabolic response in $72 \%$ and $28 \%$ of patients, respectively. Another retrospective study of 21 patients from the French IgG4-RD registry that used the same Umehara Diagnostic Criteria and similar PET/CT protocol as the prior study also demonstrated PET superiority over conventional imaging particularly for the arteries, salivary glands, and lymph nodes. ${ }^{10}$ Treatment response as assessed by PET was generally concordant with clinical assessments, with PET missing active disease in the meninges, lacrimal glands, and kidneys.

The importance of IgG4-RD imaging has been recognized by cardiology community due to the potential severity of cardiovascular complications and a significant risk associated with cardiovascular lesional biopsy. ${ }^{15}$ Arterial lesions typically show late enhancement on CECT corresponding to adventitial sclerosing inflammation $^{16}$ aiding in the diagnostic accuracy. If CECT is performed prior to FDG PET/CT, or simultaneously with FDG PET, arterial lesions can be identified on CECT followed by their metabolic assessment on PET. Yabusaki et al., reported the findings of their retrospective analysis of 37 Japanese patients with IgG4$\mathrm{RD}$ diagnosed based upon diagnostic criteria for IgG4$\mathrm{RD}$, autoimmune pancreatitis, or IgG4 Mikulicz's disease, who had undergone neck to pelvis CECT and FDG PET/low-dose CT within one hour of FDG injection. ${ }^{13}$ Active inflammation was defined as thickened arterial wall $>2 \mathrm{~mm}$ and FDG uptake $>$ blood pool reference. Aortitis was observed in $41 \%$ (15 of 37 patients) of patients, $80 \%$ (12 of 15 patients) of whom had multiple regions involved including the iliac arteries in 35\% (13/ 37 ), infra-renal abdominal aorta in $33 \%$ (12/36), thoracic aorta in $8 \%(3 / 37)$, first branches of the thoracic aorta in $8 \%(3 / 37)$, supra-renal abdominal aorta in $6 \%(2 / 36)$, and the first branches of the abdominal aorta in 5\% (2/ 37). PET parameters, maximum standard uptake value (SUV max), and target (artery of interest) to background (venous blood pool) ratio (TBR) were significantly higher in involved vessels.

FDG PET/CT using a dedicated vascular proto$\mathrm{col}^{17,18}$ is becoming a preferred method to evaluate inflammatory arterial lesions as outlined in a Position Paper of the Cardiovascular Committee of the European Association of Nuclear Medicine (EANM) on PET imaging of atherosclerosis. ${ }^{17}$ In a dedicated vascular protocol, PET acquisition is delayed from 1 to 2 hours after FDG injection to allow sufficient FDG accumulation in the arterial wall and to reduce the intensity in the 
blood. ${ }^{17,18}$ The EANM Position Paper provides recommendations on PET/CT acquisition, processing, and interpretation in order to standardize vascular protocols across institutions and to facilitate between study comparisons. Such an approach may be particularly valuable for rare diseases, such as IgG4-RD.

The present study by Shinichi et al. demonstrates novel and intriguing insights into IgG4-RD vascular inflammation. The study found that arterial activity is elevated in patients with IgG-RD relative to control subjects regardless of presence or absence of vascular complications. This observation may potentially alter the approach toward IgG4-RD risk assessment and treatment with a shift toward more aggressive management.

The investigators prospectively recruited 42 Japanese patients with suspected IgG4-RD based on clinical criteria (78\% men with a mean age of $64 \pm 8$ years). Of these, 15 patients had definite IgG4-RD according to the comprehensive diagnostic criteria by Umehara with elevated serum IgG4 and characteristic pathologic findings. Seventeen patients had possible IgG4-RD with elevated serum IgG4 but without pathologic confirmation or with negative examination. Five patients had probable IgG4-RD with positive pathologic examination but normal serum IgG. Together, the 22 patients with possible or probable IgG4-RD were diagnosed with autoimmune pancreatitis based on the International Consensus Criteria for Autoimune Pancreatitis $2011^{19}$ and were regarded as +IgG4-RD for the purpose of this study. The 5 patients with normal serum IgG4 and negative pathology were excluded. The 37 study patients who received FDG PET/CT using dedicated vascular inflammation protocol (PET scan 2 hours after injection) were age- and gender matched with 37 control patients who underwent the same protocol for other indications. Patients $<20$ years old or with acute infection or inflammation, uncontrolled or insulin-treated diabetes, malignancy, and receiving immunosuppressive therapy were excluded. The FDG activity was assessed in 6 regions, including first 3 branches from the thoracic aorta, ascending aorta, descending aorta, supra-renal abdominal aorta, infra-renal abdominal aorta, and bilateral common iliac arteries. The arterial activity in the study patients with and without vascular complications and control patients was compared using TBR with the inferior vena cava blood pool set as the reference. The separate CECT used for lesion identification was performed within 2 weeks from FDG PET/CT but had inconsistent anatomic coverage (first 3 branches from the thoracic aorta 13/37, ascending aorta 15/37, descending aorta $15 / 37$, supra-renal abdominal aorta $37 /$ 37 , infra-renal abdominal aorta 37/37, and bilateral common iliac arteries 29/37).
Common IgG4-RD-involved sites were pancreas $(86 \%)$, lymph nodes $(65 \%)$, lungs $(35 \%)$, retroperitoneum (32\%), and submandibular glands (27\%). Vascular complications were present in $32 \%$ of patients and included arteritis (enhancing wall thickening $\geq 2$ $\mathrm{mm}$ ) and aneurysms (diameter $>45 \mathrm{~mm}$ for thoracic and $>30 \mathrm{~mm}$ for abdominal, aorta), all of which occurred in the abdominal aorta. No aortic dissection or coronary involvement was observed. IgG4-RD patients had higher TBR ratio $(P<.05)$ in the descending aorta, supra-renal abdominal aorta, infra-renal abdominal aorta, and bilateral common iliac arteries relative to control subjects. A similar trend was observed in the first 3 branches from the thoracic aorta $(P=.107)$ and ascending aorta $(P=.202)$, but statistical significance was not reached possibly due to missing data caused by inconsistent CECT coverage. TBR ratio was significantly higher in the infra-renal abdominal aorta and the common iliac arteries in IgG4-RD patients with versus without vascular complications.

Although this study presents the highest quality evidence regarding the potential value of dedicated FDG $\mathrm{PET} / \mathrm{CT}$ exams for detection of vascular inflammmation in IgG4-RD patients to date, a number of limitations are present similar to prior reports. First, this study was conducted at one academic center in a small and likely homogeneous, IgG4-RD patient population and therefore, the results may not be generalizable. Secondly, a significant selection bias was likely present since PET scans were performed for a clinical indication which could result in the recruitment of sicker patients amplifying the differences between study and control subjects. Thirdly, since posttreatment scans were not performed, it is unknown if the observed differences persisted following treatment. Moreover, inconsistent CT coverage likely resulted in false-negative findings in the first 3 branches from the thoracic and ascending aorta and thus the true frequency of vascular involvement in this study could be underestimated. Lastly, although prior studies utilized Umehara's diagnostic criteria, the application seems inconsistent particularly when organ-specific criteria are incorporated in the decision matrix.

In spite of these limitations, the finding of measurable subclinical vascular reactivity in IgG4-RD patients compared to matched control patients raises the important question of whether or not this reactivity eventually leads to complications if left untreated or incompletely treated. This question can be extended to other critical organs presuming that subclinical reactivity is present and can be quantified. Implementation of a dedicated and standardized vascular FDG PET/CT protocol will enable these questions to be addressed whereby patients can be randomized to usual care (for clinically actionable vascular inflammation) versus 
escalated care (for subclinical vascular inflammation) and followed over time. The risks versus benefits of escalated treatment will have to be carefully considered in order to perform optimal patient care. However, we have to congratulate Dr. Shinichi and colleagues for laying an important foundation for further research.

In summary, Shinichi et al. conducted an informative FDG PET/CT study using a dedicated vascular protocol in patients who fulfilled specific diagnostic criteria for IgG4-RD and discovered subclinical vascular reactivity only in IgG4-RD patients compared to matched controls for which the clinical significance is currently unknown. Future studies are needed to determine the clinical and therapeutic implications of subclinical vascular inflammation in patients with IgG4RD.

\section{Disclosure}

Darko Pucar received consulting fees from Telix Pharmaceuticals and Cohere Health. Monique Hinchcliff has nothing to disclose.

\section{References}

1. Deshpande V, Zen Y, Chan JK, Yi EE, Sato Y, Yoshino T. Consensus statement on the pathology of IgG4-related disease. Mod Pathol 2012;25:1181-92.

2. Stone JH, Khosroshahi A, Deshpande V, Chan JK, Heathcote JG, Aalberse R, et al. Recommendations for the nomenclature of IgG4-related disease and its individual organ system manifestations. Arthritis Rheum 2012;64:3061-7.

3. Stone JH, Zen Y, Deshpande V. IgG4-related disease. N Engl J Med 2012;366:539-51.

4. Kasashima S, Zen Y, Kawashima A, Konishi K, Sasaki H, Endo $\mathrm{M}$, et al. Inflammatory abdominal aortic aneurysm: Close relationship to IgG4-related periaortitis. Am J Surg Pathol 2008;32:197-204.

5. Umehara H, Okazaki K, Masaki Y, Kawano M, Yamamoto M, Saeki T, et al. Comprehensive diagnostic criteria for IgG4-related disease (IgG4-RD), 2011. Mod Rheumatol 2012;22:21-30.

6. Sah RP, Chari ST. Serologic issues in IgG4-related systemic disease and autoimmune pancreatitis. Curr Opin Rheumatol 2011;23:108-13.

7. Kamisawa T, Shimosegawa T, Okazaki K, Nishino T, Watanabe H, Kanno A, et al. Standard steroid treatment for autoimmune pancreatitis. Gut 2009;58:1504-7.
8. Khosroshahi A, Wallace ZS, Crowe JL, Akamizu T, Azumi A, Carruthers $\mathrm{MN}$, et al. International consensus guidance statement on the management and treatment of IgG4-related disease. Arthritis Rheumatol 2015;67:1688-99.

9. Abraham M, Khosroshahi A. Diagnostic and treatment workup for IgG4-related disease. Expert Rev Clin Immunol 2017;13:867-75.

10. Ebbo M, Grados A, Guedj E, Gobert D, Colavolpe C, Zaidan M, et al. Usefulness of 2-[ $\left.{ }^{18} \mathrm{~F}\right]$-fluoro-2-deoxy-D-glucose-positron emission tomography/computed tomography for staging and evaluation of treatment response in IgG4-related disease: A retrospective multicenter study. Arthritis Care Res (Hoboken) 2014;66:86-96.

11. Takahashi H, Yamashita H, Morooka M, Kubota K, Takahashi Y, Kaneko H, et al. The utility of FDG-PET/CT and other imaging techniques in the evaluation of IgG4-related disease. Joint Bone Spine 2014;81:331-6.

12. Zhang J, Chen H, Ma Y, Xiao Y, Niu N, Lin W, et al. Characterizing IgG4-related disease with (1)(8)F-FDG PET/CT: A prospective cohort study. Eur $\mathrm{J}$ Nucl Med Mol Imaging 2014;41:1624-34.

13. Yabusaki S, Oyama-Manabe N, Manabe O, Hirata K, Kato F, Miyamoto N, et al. Characteristics of immunoglobulin G4-related aortitis/periaortitis and periarteritis on fluorodeoxyglucose positron emission tomography/computed tomography co-registered with contrast-enhanced computed tomography. EJNMMI Res 2017:7:20.

14. Einspieler I, Thurmel K, Pyka T, Eiber M, Wolfram S, Moog P, et al. Imaging large vessel vasculitis with fully integrated PET/ MRI: A pilot study. Eur J Nucl Med Mol Imaging 2015;42:101224.

15. Mavrogeni S, Markousis-Mavrogenis G, Kolovou G. IgG4-related cardiovascular disease. The emerging role of cardiovascular imaging. Eur J Radiol 2017;86:169-75.

16. Inoue D, Zen Y, Abo H, Gabata T, Demachi H, Yoshikawa J, et al. Immunoglobulin G4-related periaortitis and periarteritis: CT findings in 17 patients. Radiology 2011;261:625-33.

17. Bucerius J, Hyafil F, Verberne HJ, Slart RH, Lindner O, Sciagra R, et al. Position paper of the Cardiovascular Committee of the European Association of Nuclear Medicine (EANM) on PET imaging of atherosclerosis. Eur $\mathrm{J}$ Nucl Med Mol Imaging 2016;43:780-92.

18. Bucerius J, Mani V, Moncrieff C, Machac J, Fuster V, Farkouh $\mathrm{ME}$, et al. Optimizing 18F-FDG PET/CT imaging of vessel wall inflammation: The impact of $18 \mathrm{~F}-\mathrm{FDG}$ circulation time, injected dose, uptake parameters, and fasting blood glucose levels. Eur J Nucl Med Mol Imaging 2014;41:369-83.

19. Shimosegawa T, Chari ST, Frulloni L, Kamisawa T, Kawa S, Mino-Kenudson M, et al. International consensus diagnostic criteria for autoimmune pancreatitis: Guidelines of the International Association of Pancreatology. Pancreas 2011;40:352-8.

Publisher's Note Springer Nature remains neutral with regard to jurisdictional claims in published maps and institutional affiliations. 